\title{
Combination of liver enzymes, amylase and abdominal ultrasound tests have acceptable diagnostic values as an alternative test for abdominopelvic CT scan in blunt abdominal
} trauma

\author{
Reza Mosaddegh (1D' \\ Samane $\mathrm{Nabi}^{2}$ \\ Sogand Daei ${ }^{3}$ \\ Fatemeh Mohammadi (D) \\ Gholamreza Masoumi ${ }^{2}$ \\ Samira Vaziri (1D) \\ Mahdi Rezai (D) \\ 'Emergency Medicine Management \\ Research Center, Faculty of Medicine, \\ Iran University of Medical Sciences, \\ Tehran, Iran; ${ }^{2}$ Trauma and Injury \\ Research Centre, Faculty of Medicine, \\ Iran University of Medical Sciences, \\ Tehran, Iran; ${ }^{3}$ Student Research \\ Committee, Faculty of Medicine, Iran \\ University of Medical Sciences, Tehran, \\ Iran
}

This article was published in the following Dove Press journal: Open Access Emergency Medicine

Introduction: Abdominal trauma accounts for $15-20 \%$ of all-cause mortality of trauma. Abdominopelvic CT scan with intravenous contrast is considered the most accurate noninvasive diagnostic tool in detecting intra-abdominal injuries. In previous studies, rise in liver enzymes and amylase was associated with intra-abdominal injuries but the studies were not sufficient. Our aim was to assess the diagnostic values of liver enzymes and amylase for intra-abdominal injuries in blunt trauma patients.

Methods: We included blunt abdominal trauma patients who referred to three teaching hospitals in 2018. The patients who had 14 years old or more and Glasgow Coma Scale above 8 were enrolled the study if the treating physician had high index of suspicion for intra-abdominal injuries and sent the patients for abdominopelvic CT scan with intravenous contrast. Sensitivity, specificity, positive and negative predictive values are calculated for results of liver enzymes, amylase and abdominal ultrasound.

Results: Eventually, 300 patients with blunt abdominal trauma entered the study. Sensitivity, specificity, positive and negative predictive values of concurrent positive results of abdominal ultrasound, amylase and liver enzymes were 81.73 (95\% CI, 73.2-88.1), 63.78 (95\% CI, 65.36-70.61), 58.38 (95\% CI, 56.36-70.61) and 84.89 (95\% CI, 77.6-90.19), respectively.

Conclusion: Considering findings of the present study, the combination of liver enzymes, amylase and abdominal ultrasound results can be an alternative method for detecting intraabdominal injuries in patients in whom treating physicians have limitations such as overweight, instability of hemodynamic and lack of CT scan facility.

Keywords: blunt abdominal trauma, liver enzymes, amylase, intra-abdominal injury

\section{Introduction}

While trauma is a leading cause of mortality around the globe, WHO statistics suggest that more than 1.2 million people die of traumas every year and another 50 million suffer morbidities. ${ }^{1,2}$ Trauma is the fourth cause of mortality in Iran and the leading cause of death in the young population. ${ }^{3}$ Abdominal trauma also accounts for $15-20 \%$ of deaths from trauma. Mortalities caused by abdominal trauma can be either early death (due to hemorrhage) or late death (due to infection and sepsis). ${ }^{4}$ The most common mechanism of blunt trauma is a car accident. The mechanisms of
Correspondence: Mahdi Rezai Emergency Medicine Management Research Center, Hazrat Rasool Hospital, Niayesh Street, Sattarkhan Street, Tehran, Iran

Tel +98912 I38 1048

Email rezaei.m@iums.ac.ir 
blunt abdominal trauma include crush injury, compression injury and acceleration and deceleration injuries. ${ }^{5}$ The mortality risk of blunt abdominal trauma is higher than penetrating trauma, which could be attributed to the difficulties in the diagnosis of the injury, the severity of trauma and other comorbidities. ${ }^{5}$ The most reliable signs and symptoms in conscious patients are pain, tenderness and signs of stimulation of the peritoneum. The reliability of clinical examinations decreases with a decrease in patient's consciousness. ${ }^{5}$ Moreover, the hollow viscera injury refers to a set of symptoms such as hemorrhage and stimulation of the peritoneum. Hemorrhage may be limited and invisible in the physical examinations. Stimulation of the peritoneum may also be painful but the signs and symptoms are not manifested immediately. ${ }^{4}$ Although there are several methods of diagnosing intraabdominal injuries, there is a lack of evidence that confirm the results. Therefore, a combination of conservative clinical examinations, assessment of the pathology mechanism and selection of a conservative diagnostic approach is employed. ${ }^{4}$ Physicians often use a combination of radiology and ultrasonography (US) to perform a primary assessment of patients suffering from high-energy traumas or trauma patients with low levels of consciousness. In this regard, FAST (focused assessment with US for trauma) is a useful modality for the primary assessment of trauma patients, which does not make any difference in the resuscitation maneuvers. ${ }^{6}$ FAST mainly serves the rapid diagnosis of the fluid in the peritoneum in patients suffering from blunt abdominal trauma and low blood pressure. Its other advantages include its high accuracy and speed, noninvasiveness, repeatability and being replaceable. ${ }^{6}$ Although the sensitivity of FAST in the diagnosis of the fluid in the peritoneum is higher than a CT scan, a CT scan helps decide on the treatment (surgical or nonsurgical) with a positive FAST result. ${ }^{7}$ As compared to a CT scan, the most important pitfall of FAST is its inability to accurately diagnose the origin of the fluid in the peritoneum. ${ }^{4}$ An abdominopelvic CT scan with intravenous contrast materials is the noninvasive diagnosis standard for abdominal injuries. The most important advantage of this method is determining the location and severity of the injuries. However, there are two difficulties in the use of this method, which include the use of radiation and the need for emergency departure. ${ }^{4}$ Experimental tests: an increase in lipase and amylase improves the injury, but it is not definitive and further assessments are required. Besides, the results from the liver function tests do not make a distinction between serious and minor injuries. Therefore, a combination of FAST and liver function tests can be used in the primary assessment of pediatric trauma to avoid a CT scan. ${ }^{5}$ Given the mentioned constraints upon the clinical examination, FAST and CT scan methods for the diagnosis of blunt abdominal trauma and the economic burden shouldered by the health and treatment sector and the emergency sector, it is necessary to quickly diagnose and facilitate the accurate diagnosis of these patients.

Some of the previous studies have revealed that increased serum transaminase might cause blunt hepatic trauma. ${ }^{8}$ It has also been proven that transaminase levels can be as valuable as FAST for the clarification of the disposition of patients with hepatic injuries. ${ }^{9}$ Therefore, the present research was an attempt to unravel the relationship between amylase and liver enzyme levels in patients suffering from blunt abdominal trauma. Similar studies have been carried out on children due to the inefficiency and inadequacy of FAST, the constraints on clinical examination and the risk of radiation in CT scans $(2-4 \%){ }^{5}$ However, extensive research has not been conducted on the application of these tests in clarifying the disposition of adult patients. The present research is, therefore, a prospective study on the diagnostic value of the combination of amylase and liver enzyme experimental tests and US for the prediction of intra-abdominal injuries in adults suffering from blunt abdominal trauma. The ultimate goal of this research is to determine whether it is possible to reduce the need for CT scans in patients with blunt abdominal trauma by dint of US and liver enzymes and amylase tests.

\section{Methods}

\section{Study design and ethics}

This research was carried out as an observational-analytical cross-sectional study on patients in three academic hospitals affiliated with Iran University of Medical Sciences (IUMS), namely Rasool Akram, Firuzgar and Haft-e Tir hospitals. This study was also conducted in accordance with Helsinki Accords, and thus the informed consent of all patients meeting the inclusion criteria was obtained. Actually, all patients signed the written informed consent. It is noteworthy that none of the patients received extra radiation in the course of this project and only abdominopelvic CT scans were carried out due to the high likelihood of intra-abdominal injuries. In addition, no additional blood sample was collected from the samples 
and no extra expense was imposed on the patients in the AST, ALT and amylase tests. Approval was obtained from the ethics committee of IUMS.

\section{Study population and setting}

The research population consisted of patients aged over 18 years with blunt abdominal trauma and GCS $>8$, who were clinically suspected of intra-abdominal injuries based on physical examinations. Thus, all had clinical indication for abdominopelvic CT scanning. The study was performed in three academic emergency departments of Rasoul Akram, Firuzgar and Haft-e Tir hospitals. Patients suffering from penetrating trauma, pregnant patients, patients suffering from spinal cord injuries and patients in unstable hemodynamic conditions were excluded from this research. The calculated sample size was 246 according to the following formula, but 300 patients were studied to increase the accuracy and precision of the research results.

Sample size based on sensitivity: $\frac{Z^{2} \times P(1-P)}{d^{2}}$

Z: 1.96 , d: 0.05, P: Sensitivity: 80

\section{Research methodology}

FAST US and abdominopelvic CT scans with intravenous contrast materials were carried out for all patients suffering from blunt abdominal trauma that met the inclusion criteria. The CT scan machines were Philips, brilliance CT Scan 16 Slice (Philips, Hospital \& Health CareAmsterdam, NoordHolland). The CT scanner machines acquired images with a slice thickness of $2.5 \mathrm{~mm}$. For each patient, injection of 50 $75 \mathrm{~mL}$ non-ionic contrast (iopromide, Ultravist; Bayer Schering Pharma AG, Berlin, Germany) was performed by using a $60-\mathrm{mL}$ irrigation syringe. Blood samples were also collected for the routine tests. After making arrangements with the laboratory, the Aspartate Aminotransferase (AST), Alanine Aminotransferase (ALT) and amylase levels were measured using the collected blood samples and Pars Azmun kits (Pars Azmun Co., Medical Devices, Karaj, Alborz, Iran). Afterward, based on the laboratory kits, results higher than $40 \mathrm{U} / \mathrm{I}, 40 \mathrm{U} / \mathrm{I}$ and $100 \mathrm{U} / \mathrm{I}$ were considered to be indicative of abnormal AST, ALT and amylase levels, respectively. FAST US was carried out by an attending emergency physician, and the abdominopelvic CT scan reports were prepared by an attending radiologist. In the end, the researchers compared the CT scan, US and AST, ALT and amylase experimental test results. All patients were followed-up within one week of admission to track their subsequent visits to the other centers or the aforesaid centers for intra-abdominal injuries from traumas.
The study variables included age, gender and amylase, AST and ALT levels. The FAST and CT scan results are reported in the following section.

\section{Statistical analysis}

Data analysis was carried out in SSPSS v.22 (IBM, Armonk, NY, USA). The numerical variables were expressed in terms of mean and standard deviation (Mean $\pm \mathrm{SD}$ ), and qualitative variables were expressed in terms of frequency and frequency percentage. The qualitative data were analyzed through a chi-squared test, while a student's $t$-test (or its nonparametric equivalent) was conducted to analyze the quantitative data. Finally, the diagnostic accuracy, sensitivity, specificity and positive and negative predictive values of the US, CT scan and AST, ALT and amylase tests were calculated and reported. Significance level was considered below 0.05 .

\section{Results}

A total of 300 patients suffering from blunt abdominal trauma were included in this study. FAST, abdominopelvic CT scans and amylase, AST and ALT tests were carried out for all patients. The average serum AST, ALT and amylase levels were $56.87 \pm 63.69,51.94 \pm 63.10$ and 65.35 \pm 53.41 , respectively. According to references values, $38 \%$, $35.33 \%$ and $10.66 \%$ of the patients had showed AST, ALT and amylase levels higher than normal, respectively. Injuries to a specific organ were mentioned in the CT scan reports of $62.26 \%$ of patients with high levels of ALT. Moreover, $88.8 \%$ of patients suffering from spleen injuries had high serum ALT levels, while the serum ALT levels in patients suffering from liver, kidney, pelvic and gastrointestinal (GI) system injuries were higher than normal values in $68.05 \%, 25 \%, 16.6 \%$ and $0 \%$, respectively. A hundred percent of patients with concurrent spleen and hepatic injuries showed high serum ALT levels $(P<0.001)$ (Table 1).

Also, injuries to at least one organ were mentioned in the CT scan reports of $60.52 \%$ of patients with high levels of AST. We showed that $77.7 \%$ of patients suffering from spleen injuries had high serum AST levels, while the serum AST levels in patients suffering from liver, kidney, pelvic and GI system injuries were higher than normal values in $73.61 \%, 25 \%, 16.6 \%$ and $0 \%$, respectively. A hundred percent of patients with concurrent spleen and hepatic injuries showed high serum AST levels too $(\mathrm{p}<0.001)$ (Table 1). 
Table I The association between ALT, AST and amylase levels and US results with CT scan reports

\begin{tabular}{|c|c|c|c|c|}
\hline CT scan result & High ALT & High AST & High amylase & Abnormal US \\
\hline Spleen injury & 88.8 & 77.7 & 0 & 77.7 \\
\hline Hepatic injury & 68.5 & 73.61 & 27.77 & 36.11 \\
\hline Renal injury & 25 & 25 & 0 & 83.3 \\
\hline Intra-pelvic injury & 16.6 & 16.6 & 0 & 55.5 \\
\hline GI tract injury & 0 & 0 & 0 & 100 \\
\hline Concurrent hepatic and spleen injuries & 100 & 100 & 33.33 & 100 \\
\hline Normal & 21.62 & 24.32 & 94.5 & 4.86 \\
\hline
\end{tabular}

Abbreviations: ALT, aminotransferase; AST, aspartate aminotransferase; US, ultrasonography; GI, gastrointestinal.

In the CT scan reports of $65.62 \%$ of the patients showing high serum amylase levels, a specific organ injury was reported, while the serum levels of amylase were high in $27.7 \%$ of the patients with reports of hepatic injuries. None of the patients with spleen, liver, kidney and GI tract injuries had high levels of amylase, and in $33.33 \%$ of patients, suffering from concurrent spleen and hepatic injuries the amylase level was high $(P<0.001)$ (Table 2$)$.

In $92.1 \%$ of patients with abnormal FAST exam, injury to a specific organ was reported in CT scan, while $83.3 \%$ of the patients suffering from renal injuries had abnormal FAST results. The percentages of abnormal FAST exam in patients suffering from spleen, liver and intra-pelvic injuries were $77.7 \%, 36.11 \%$ and $55.5 \%$, respectively. A hundred percent of patients with GI tract injuries and a hundred percent of patients with concurrent spleen and hepatic injuries had abnormal US results $(\mathrm{p}<0.001)$ (Table 2).

As compared to $\mathrm{CT}$ scan results (standard criterion), the sensitivity, specificity, positive and negative predictive values, and accuracy of the serum AST, ALT and amylase levels and US results for the diagnosis of organ damages in blunt abdominal trauma are introduced in Table 2.

The sensitivity, specificity, positive predictive value and negative predictive value of AST, ALT and amylase and US when their results considered simultaneously were 81.73 (95\% CI 73.2-88.1), 63.78 (95\% CI 56.36-70.61), 58.38 (95\% CI 50.35-60.01) and 84.89 (95\% CI 77.6090.19), respectively (Table 2 ).

A total of 13 patients were subjected to laparotomy, and the US results of all 13 of them were abnormal. Besides, $100 \%$ and $92.30 \%$ of these patients had high ALT and AST levels, respectively $(\mathrm{p}<0.001)$.

Three patients were diagnosed with concurrent spleen and hepatic injuries, and the levels of serum AST and ALT increased in all three of them. All of these patients were also subjected to laparotomy. Only one of the 13 patients subjected to laparotomy showed a high level of amylase $(\mathrm{p}=0.58)$.

Six of the 9 patients with spleen injuries and 4 of the 72 patients with hepatic injuries were subjected to laparotomy. Of the 6 patients suffering from spleen injuries, 5 of them had high serum AST levels, and the AST level was normal in only one patient, who suffered from spleen injuries and was subjected to laparotomy $(\mathrm{p}<0.001)$. Of the 6 patients suffering from spleen injuries who underwent laparotomy, all six of them had high serum ALT levels $(\mathrm{p}<0.001)$. Of the 4 patients who were suffering from hepatic injuries and were subjected to laparotomy, all four of them had high serum AST and ALT levels $(\mathrm{p}<0.001)$.

Of the nine patients who were diagnosed with spleen injuries based on the CT scan reports, 2 of them had abnormal US results and normal AST levels, while the other two had normal US exams and high serum AST levels $(\mathrm{p}<0.001)$. Moreover, of the 72 patients diagnosed with hepatic injuries based on CT scan results, 14 of them had normal US exams and normal AST levels, and 21 had abnormal US results and high serum AST levels $(\mathrm{p}<0.001)$. Of the 185 patients with normal CT scan results, 140 patients had normal serum AST levels and 45 had high serum AST levels. Of these 45 patients, 44 had normal US exams $(\mathrm{p}<0.001)$.

Of the 9 patients diagnosed with spleen injuries by CT scans, 6 had normal US exams and high levels of ALT $(\mathrm{p}<0.001)$, and of the 72 patients suffering from hepatic injuries based on CT scan results, 30 had normal US results and high ALT levels $(p<0.001)$. Among the 185 patients with normal CT scan results, 145 patients had normal ALT levels and 40 showed high serum ALT levels. Of the latter 40 patients, 39 had normal US exams $(p<0.001)$. A total of 72 patients were also diagnosed with hepatic injuries through CT scans. Of these patients, 
Table 2 Sensitivity, specificity, positive and negative predictive values of ALT, AST, amylase and US in detecting abdominal injuries in blunt abdominal trauma

\begin{tabular}{|l|l|l|l|l|l|}
\hline & Sensitivity & Specificity & PPV & NPV & Accuracy \\
\hline AST & 60 & 75.67 & 60.52 & 75.26 & 69.66 \\
ALT & 57.39 & 78.37 & 62.26 & 74.44 & 70.33 \\
Amylase & 18.26 & 94.05 & 65.26 & 64.92 & 65 \\
US & 49.56 & 95.13 & 86.36 & 75.21 & 77.66 \\
\hline
\end{tabular}

Abbreviations: ALT, aminotransferase; AST, aspartate aminotransferase; US, ultrasonography; GI, gastrointestinal.

20 had high levels of amylase, and 11 of these 20 patients had normal US results $(\mathrm{p}=0.049)$.

All of the patients who were subjected to laparotomy because of hepatic and spleen injuries had abnormal US results. Five of the 6 patients who had spleen injuries and were subjected to laparotomy showed high AST levels, but all patients with hepatic injuries and concurrent hepatic and spleen injuries had high AST levels $(\mathrm{p}=0.053)$. As regards ALT, all patients subjected to laparotomy had high ALT levels.

The average ALT levels in the laparotomy group (range: 73-660) and nonsurgical group (range: 8-298) were 167.62 \pm 154.18 and $46.70 \pm 50.29$, respectively $(\mathrm{p}<0.001)$. The average AST levels in the laparotomy group (range: 30-507) and nonsurgical group (range: 5-467) were $150.5 \pm 112.3$ and $52.64 \pm 57.44$, respectively $(\mathrm{p}<0.001)$. The average amylase levels in the laparotomy group (range: 57-139) and the nonsurgical group (range: 17-654) were also $86.15 \pm 17.05$ and 63.96 \pm 54.09 , respectively $(\mathrm{p}=0.03)$.

In this study, 185 patients had normal CT scan results and were discharged. These 185 patients were followed-up within one week of their admission to the treatment centers for their subsequent visits to these centers or other centers due to the complications of trauma. However, none of them visited in any treatment center.

\section{Discussion}

According to the results from this study, when a CT scan of blunt abdominal trauma cases is not available due to hemodynamic instability, unavailability of CT scan services or physical limitations (high weight), the results from the AST, ALT and amylase tests and FAST US can be used instead of abdominal CT scans with intravenous contrast to identify the cases that are in need of laparotomy due to blunt abdominal trauma. Moreover, the combination of AST, ALT and amylase tests and FAST US offers a sensitivity and specificity of higher than $81.73 \%$ and $63.78 \%$, respectively. However, the amylase test is fruitless and ineffective on its own.
Abdominal trauma is increasing daily due to the increased number of vehicles, resulting in death, hospitalization or long-term disabilities in the first four decades of life. ${ }^{10}$ Liver is the fourth most vulnerable organ that is injured in abdominal traumas, ${ }^{11}$ and hepatic injuries clearly increase mortality and morbidity rates. ${ }^{12}$ Moreover, hepatic injuries are a challenge to physicians and are linked to a mortality rate of above $50 \%{ }^{12}$ The increased level of transaminases in blunt hepatic trauma can be a direct indication of the severity of hepatic injuries. The rate of laparotomy is also higher among patients who have blunt hepatic traumas, AST levels higher than $419 \mathrm{U} / \mathrm{L}$ and ALT levels higher than $498 \mathrm{U} / \mathrm{L}$. Also, it is reported that the measurement of transaminase levels is more effective than FAST in determining the laparotomy indication. ${ }^{13}$ Bilgic and colleagues performed a retrospective study on 96 patients with traumatic liver injury. They found that the circulating AST and ALT levels were significantly higher in patient with higher grade of liver injury (3-5). Their finding showed that ALT $\leq 76 \mathrm{U} / \mathrm{L}$ and AST $<130 \mathrm{U} / \mathrm{L}$ are predictive of low-grade liver injury, and patients with serum liver levels below these levels can be managed conservatively. ${ }^{12}$ In another study, receiver operating characteristic (ROC) curve analysis was applied to determine the optimal cut-off values for AST and ALT in diagnosing blunt liver injury. They identified AST $\geq 109 \mathrm{U} / 1$ and ALT $\geq 97 \mathrm{U} / 1$ as optimal cut-off values in predicting the presence of liver injury. Based on mentioned values, AST $\geq 109 \mathrm{U} / 1$ had a sensitivity of $81 \%$, a specificity of $82 \%$, a PPV of $32 \%$ and a NPV of $98 \%$. The corresponding values for ALT $\geq 97 \mathrm{U} / 1$ were $78 \%, 88 \%, 41 \%$ and $98 \%$, respectively, and for the combination of AST $\geq 109 \mathrm{U} / 1$ and/or ALT $\geq 97 \mathrm{U} / 1$ were $84 \%, 81 \%, 32 \%$ and $98 \%$, respectively. ${ }^{14}$

The results from this research suggest that the levels of AST and ALT are above normal in more than $60 \%$ of the patients suffering from spleen or hepatic injuries following blunt abdominal traumas. Interestingly, in all patients with concurrent spleen and hepatic injuries, the levels of these enzymes increase. The serum AST and ALT levels in most 
patients with hepatic traumas also increase. ${ }^{12}$ In the animal and human studies, the levels of these enzymes increased a few hours following the blunt abdominal trauma, which was linked to the increase in the severity of hepatic injuries. $^{15}$ The increased level of these enzymes can be a sensitive marker of involvement of intra-abdominal organs in blunt traumas. ${ }^{16}$ Besides, the combination of the results from the AST, ALT and amylase tests and FAST US seems to improve all statistical parameters, especially sensitivity.

\section{Conclusion}

The results from our research and other studies imply that the sensitivity and specificity of each of the aforementioned tests are not enough for rejecting post-trauma abdominal injuries, because the increase in each enzyme seemingly originates from injuries to a specific organ.

As seen in Table 2, the sensitivity of AST and ALT was higher than amylase and US, whereas the specificity of amylase and US was higher than AST and ALT. Since the overarching goal of this research was to clarify the disposition of patients suffering from blunt abdominal trauma and reject or approve the injuries, the sensitivity of the results was of higher importance. The integration of all test results also led to a sensitivity of $81.73 \%$, which was acceptable.

\section{Acknowledgment}

We thank Research and Development Center of Firoozgar Hospital for supporting us in data collection and performing this study.

\section{Disclosure}

The authors report no conflicts of interest in this work.

\section{References}

1. Word Health Organization, Global status report on road safety 2015. Supporting a Decade of Action Geneva. WHO press; 2015.

2. Pfeifer R, Teuben M, Andruszkow H, Barkatali BM, Pape HC. Mortality pattern in patients with multiple trauma: a systematic review of autopsy studies. PLoS One. 2016;11(2):e0148844. doi:10.1371/journal.pone. 0148844
3. Herfatkar MR, Mobayen MR, Karimian M, Rahmanzade F, Baghernejade S, Baghai I. Serial clinical examination of 100 patients treated for anterior abdominal wall stab wound: a cross sectional study. Trauma Mon. 2015;20(4):e24844. doi:10.5812/traumamon. 24844

4. Tintinalli JE, Stapczynski JS, Ma OJ, Yealy DM, Meckler GD, Cline DM. Tintinalli's Emergency Medicine, A Comprehensive Study Guide. 8th ed. 2016:1681-1772.

5. Marx JA, Hockberger RS, Walls RM. Rosen's Emergency Medicine, Concept and Clinical Practice. 8th ed. 2013:287-511.

6. Zhao DW, Tian M, Zhang LT, et al. Effectiveness of contrast enhanced ultrasound and serum liver enzyme measurement in detection and classification of blunt liver trauma. J Int Med Res. 2017;45:170-181. doi:10.1177/0300060516678525

7. Dammers D, El Moumni M, Hoogland II, Veeger N, Ter Avest E. Should we perform a FAST exam in haemodynamically stable patients presenting after blunt abdominal injury: a retrospective cohort study. Scand J Trauma Resusc Emerg Med. 2017;25:1. doi:10.1186/s13049-017-0455-0

8. Prasad P, Acharya A, Ellikunnel Vithon G, Kumar GNM. Evaluating the role of liver enzymes as predictors of severity of liver injury in patients with blunt abdominal trauma. Int $J$ Res Med Sci. 2017;5(6):2462-2467. doi:10.18203/2320-6012. ijrms20172429

9. Bruhn PJ, Østerballe L, Hillingsø J, Svendsen LB, Helgstrand F. Posttraumatic levels of liver enzymes can reduce the need for CT in children: a retrospective cohort study. Scand J Trauma Resusc Emerg Med. 2016;24:104. doi:10.1186/s13049-016-0297-1

10. Maske AN, Deshmukh SN. Traumatic abdominal injuries: our experience at rural tertiary care center. Int Surg J. 2016;3(2):543-548. doi:10.18203/2349-2902.

11. Slotta JE, Justinger C, Kollmar O, Kollmar C, Schäfer T, Schilling MK. Liver injury following blunt abdominal trauma: a new mechanism-driven classification. Surg Today. 2014;44(2):241-246. doi:10.1007/s00595-013-0515-7

12. Bilgic I, Gelecek S, Akgun AE, Ozmen MM. Predictive value of liver transaminases levels in abdominal trauma. Am J Emerg Med. 2014;32 (7):705-708. doi:10.1016/j.ajem.2014.03.052

13. Koca B, Karabulut K, Ozbalci GS, et al. Is it possible to use transaminases for deciding on surgical or non-operative treatment for blunt liver trauma? Wien Klin Wochenschr. 2015;127(23-24):954-958. doi:10.1007/s00508-015-0708-8

14. Koyama T, Hamada H, Nishida M, Naess PA, Gaarder C, Sakamoto T. Defining the optimal cut-off values for liver enzymes in diagnosing blunt liver injury. BMC Res Notes. 2016;9:41.

15. Ritchie AH, Williscroft DM. Elevated liver enzymes as a predictor of liver injury in stable blunt abdominal trauma patients: case report and systemic review of the literature. Can J Rural Med. 2006;11:283287.

16. Sahdev P, Garramone RR, Schwartz RJ, Steelman SR, Jacobs LM. Evaluation of liver function tests in screening for intraabdominal injuries. Ann Emerg Med. 1991;20:838-841. doi:10.1016/s01960644(05)81422-2
Open Access Emergency Medicine

\section{Publish your work in this journal}

The Open Access Emergency Medicine is an international, peerreviewed, open access journal publishing original research, reports, editorials, reviews and commentaries on all aspects of emergency medicine. The manuscript management system is completely online and includes a very quick and fair peer-review system, which is all easy to use. Visit http://www.dovepress.com/testimonials.php to read real quotes from published authors. 